\title{
Enigma of Debonding
}

\author{
Dr Chandrika Dubey,' Dr Amit Prakash,,2 Dr Anil Sharma,3 Dr Upendra Jain \\ 'PG Resident, 2.3Reader, ${ }^{4}$ Professor, Dept of Orthodontics \\ Peoples College of Dental Sciences, Bhopal, India
}

Correspondence: Dr Amit Prakash; Email: amitprakash3o@gmail.com

\section{ABSTRACT}

This article overviews the effects of debonding procedures on tooth enamel and reviews a variety of mechanical methods that have been designed to achieve satisfactory composite removal with minimal damage to the enamel surface. The orthodontic procedures starting from etching, priming to debonding cause irreversible enamel loss. The orthodontic treatment should ensure benefit to the patient; the advantages of treatment should far outweigh any adverse sequelae that might cause by the treatment. To prevent, minimize and manage the possible adverse effects of debonding mechanics, the clinician should be aware of the problems that may occur during the treatment procedures.

Keywords: ceramic brackets, debonding, enamel, metal brackets

\section{INTRODUCTION}

After completion of fixed orthodontic treatment, one of the major concerns of orthodontist is to bring the enamel to the previous form. It is a major issue as the enamel undergoes etching; priming and composite application causing minor surface cracks which actually increase the bond strength of the enamel but can be a causative factor for the enamel to become weak. For many decades, the most popular bonding system in orthodontics has been based on the acid-etching technique introduced by Buonocore in $1955^{1}$ and modified for orthodontic purpose by Newman ${ }^{2,3}$ and Retief et a ${ }^{3}$ during 1960's. The essential step in this technique is the preconditioning of enamel surface with $37 \%$ orthophosphoric acid solution or gel for approximately 30 seconds to dissolve the enamel minerals. Rinsing with water, priming, and bonding the attachments with adhesive follow the etching step. Research has shown that bonding material may penetrate relatively deep into the etched enamel surface. ${ }^{4}$ So, there is a risk that resin material may persist in the enamel after debonding, and these resin remnants may discolor in due time. ${ }^{5}$

\section{Bonding procedure and enamel surface}

Cleaning: Typically, the pumicing process results in enamel loss of 5-14 $\mu \mathrm{m}$, depending on the duration and type of instrument used to clean the surface. ${ }^{6}$ A brush removes more tissue than rubber cup $(6.90 \mu \mathrm{m}$ and 14.38 $\mu \mathrm{m}$ respectively); which are more than abrasive paste or slurry used. Both procedures also induce scratches in the surface (Figure 1).6.7

Etching: The modern bonding systems for resin-based materials are based on micromechanical retention principle. To achieve this, an acid, generally $37 \%$ orthophosphoric acid is applied for 15-30 seconds to clean the surface and to dissolve the minerals. The enamel etched with phosphoric acid shows macroscopically
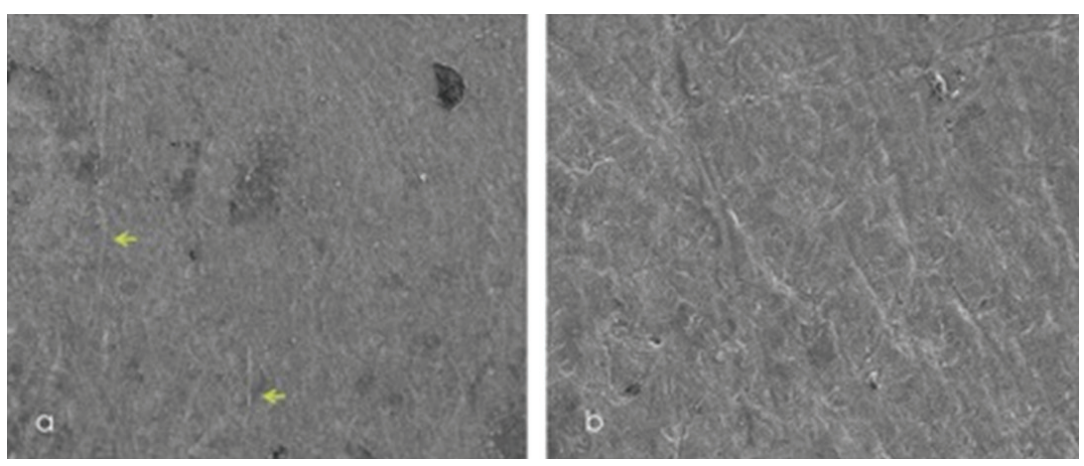

Figure 1: (a) Enamel specimen with scratches and irregularities at 250X magnification after polishing with rubber-cup for 30 seconds; (b) Same specimen at 1000X magnification 

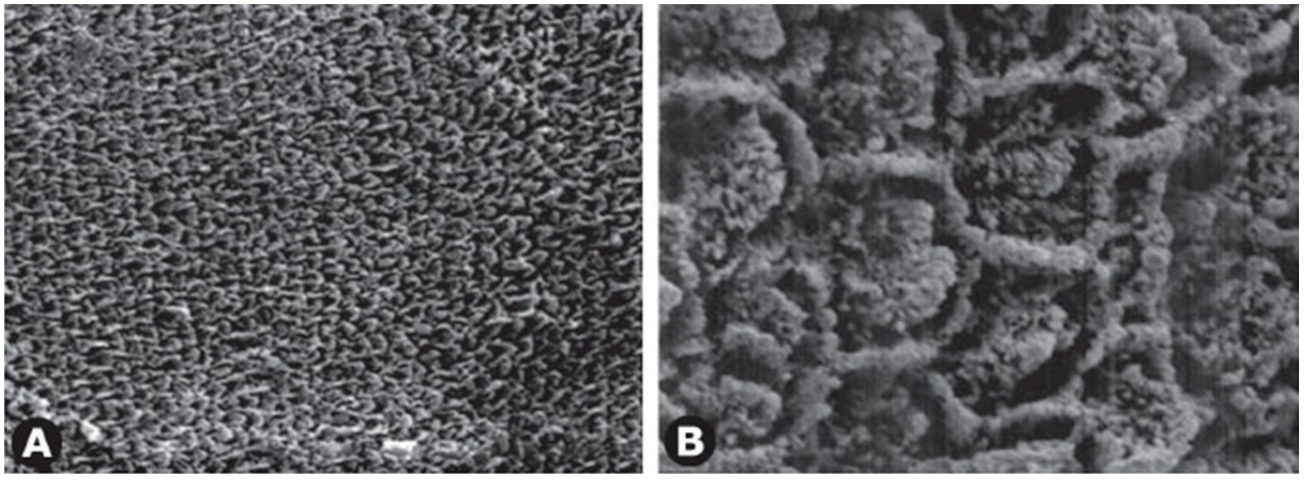

Figure 2: SEM photomicrograph of enamel surface etched with $37 \%$ ortho phosphoric acid for 30 seconds. Areas of prism removal at the core can be appreciated whereas peripheral prismatic material is intact

white, frosty appearance. In ESEM, the quality of etching varies from well-etched areas with prisms protruded in "honey comb pattern" with few small scratches to areas where the enamel is badly affected with many scratches. ${ }^{8}$ Studies evaluating composite-to-enamel bond strength obtained with self-etching adhesive systems reveal the values of 20-30 MPa, which are at the same range as that reported for enamel etched with phosphoric acid (Figure 2). ${ }^{9}$

\section{METHODS OF DEBONDING}

Various methods of debonding described in literature are:

1. Mechanical Methods: Using pliers or wrenches. ${ }^{10}$

2. Ultrasonic Method: Useing special tips; electrothermal device transmit heat to the adhesive through the bracket, air pressure impulse devices which is common for crown removal in prosthodontics, diamond burs to grind the brackets off the tooth surface. It minimizes the potential for bracket failure as well as the trauma to the enamel surface during debonding..$^{11,12}$

3. Laser Heating: Laser energy degrades the adhesive resin used to bond brackets by thermally softening it.

4. Electro-thermal Method: Overcomes the problems of bracket failure, enamel damage and high forces when debonding orthodontic brackets. The electrothermal debracketing instrument transfers heat through the bracket, allowing bond failure at the bracket-adhesive interface as the heat deforms the adhesive. ${ }^{13}$

5. Grinding: Grinding the brackets off the tooth surface by using diamond burs. The gross removal of residual composite left on the enamel surface after grinding of bracket is thought to be best accomplished with a tungsten carbide bur. Ulusoyl ${ }^{11}$ and Ryf ${ }^{12}$ stated that this method using different rotary finishing instruments is laborious and is associated with subsequent enamel damage and the loss is up to $19.2 \mu \mathrm{m}$ of enamel.

\section{Enamel surface after debonding}

Safe debonding techniques aim to break the bond between the bracket base and adhesive rather than between the adhesive and enamel surface. The residual material must be removed and the enamel surface should be polished to avoid discoloration and plaque accumulation. Enamel color alterations may occur because of the irreversible penetration of resin tags into the enamel structure at the depths reaching $30-50 \mu \mathrm{m}$ or more (Figure 3). ${ }^{14}$

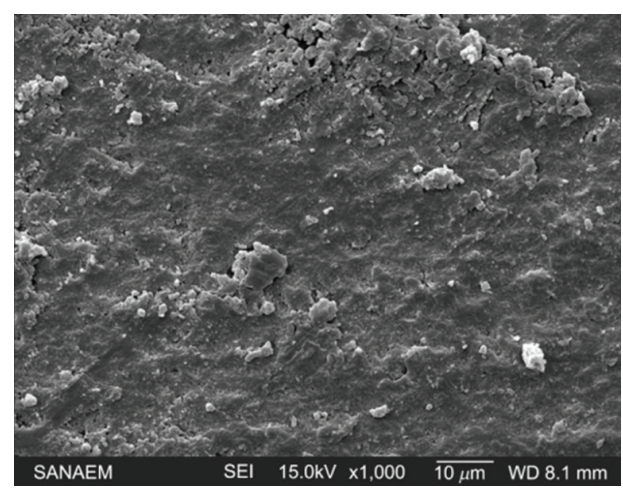

Figure 3: Photomicrograph of the acid-treated enamel surface after debonding. The entire enamel surface is coated with resin.

\section{Debonding metal brackets}

Usually metal brackets can be debonded relatively easily by applying force that peels the bracket base away from the tooth. Most often, such forces cause bond failure at the adhesive-bracket interface and most of the adhesive remain on the enamel surface after debonding. To reduce the rate of irreversible enamel surface damage, following methods of debonding have been suggested:

1. Conventional methods using pliers

2. Ultrasonic method using special tips

3. Electrothermal method using apparatus that transmits heat to the adhesive through the bracket. ${ }^{15,16}$ 


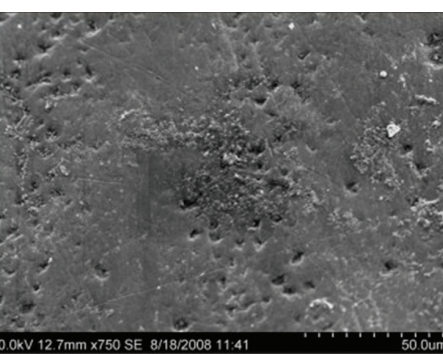

Figure 4: Enamel surface after debonding stainless steel bracket
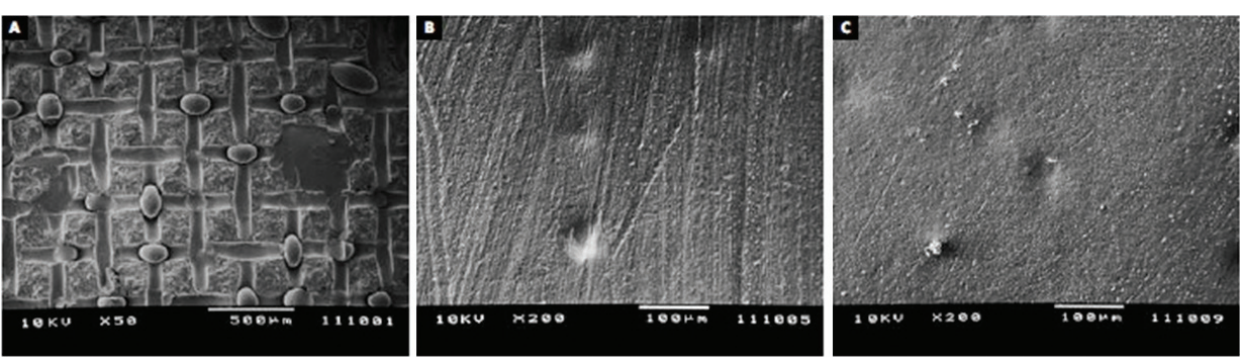

Figure 5: Photomicrographs of: (A) debonding with straight debonding plier

(B) removal of adhesive remnants with long adhesive remnant plier

(C) after polishing with rubber cup
Although all three methods can be used successfully to debond brackets; the use of pliers to apply a shear or tensile force on the bracket is perhaps the most convenient method and continues to be the most popular method used for debonding brackets. Original Method was to place the tips of a twin beaked plier against the mesial and distal edges of the bonding base and cut the bracket off between the tooth and the base. Gentler technique is to squeeze the bracket wings mesiodistally and lift the bracket off with a peel force. This is particularly useful for brittle, mobile, or endodontically treated teeth. However, the Recommended Technique is by using a peeling force, which is most effective in breaking the adhesive bond. The peeling force creates peripheral stress concentration that cause bonded metal brackets to fall at low force values. The break occurs at adhesive-bracket interface, leaving the adhesive remnants on enamel (Figure 4, 5). ${ }^{17}$

\section{Debonding ceramic brackets}

Because of the difference in bracket chemistry and bonding mechanisms, various ceramic brackets behave differently on debonding. ${ }^{17}$ Some studies reported no enamel damage when debonding ceramic brackets using appropriate pliers: ${ }^{18}$ other studies reported increased enamel cracks or crack length following debonding. Bishara reported that $18 \%$ of teeth had an increased number or severity of enamel cracks following the debonding of ceramic brackets, ${ }^{19,20}$ Preferred mechanical

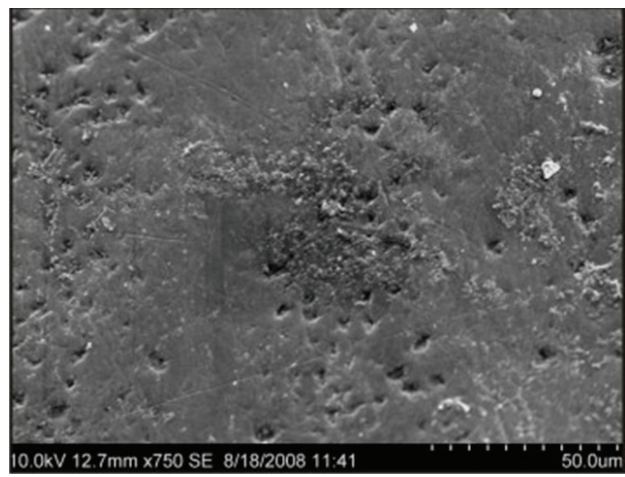

Figure 6: Enamel surface after debonding ceramic bracket debonding is to lift the brackets off with peripheral force application, same as metal brackets. Recently introduced ceramic brackets have a mechanical lock base and a vertical slot that splits the bracket by squeezing. Separation occurs at the bracket adhesive interface, which cause little risk of enamel fracture. ${ }^{21,22}$ Thermal debonding and Lasers have the potential to be less traumatic and less risky for enamel damage, but these techniques are still at the introductory stage. (Figure 6)

\section{Enamel cracks}

Enamel cracks occurring as split lines in the enamel are common but generally over-looked as they are difficult to detect unless transillumination techniques are used. The prevalence of enamel cracks after debonding has been found as high as 50\%, with vertical cracks being the most common. There is a clear correlation between enamel cracks and debonding forces. Frequent appearance of horizontal cracks after debonding may indicate improper bonding/debonding techniques. ${ }^{23}$ Zachrisson et al discussed the occurrence of cracks in debonded, debanded and orthodontically untreated teeth. He used fiberoptic light technique and examined more than 3000 teeth in 135 adolescents. He found vertical cracks were more common in more than $50 \%$ of teeth studied, but individual variance was great. He observed few horizontal and oblique cracks. The most noticeable cracks were on maxillary central incisors and canine. ${ }^{24}$ (Figure 7)

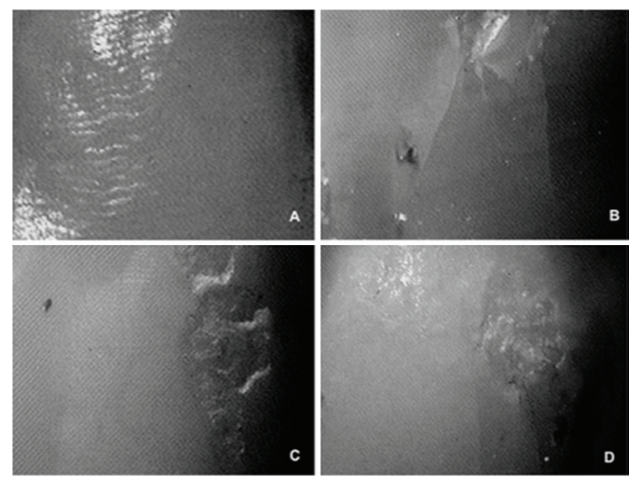

Figure 7: Photomicrographs of surface enamel: (A) no damage; (B) damage in the form of a crack; (C) damage in the form of tear-outs; (D) damage in the form of tear-outs and cracks. 
a

b

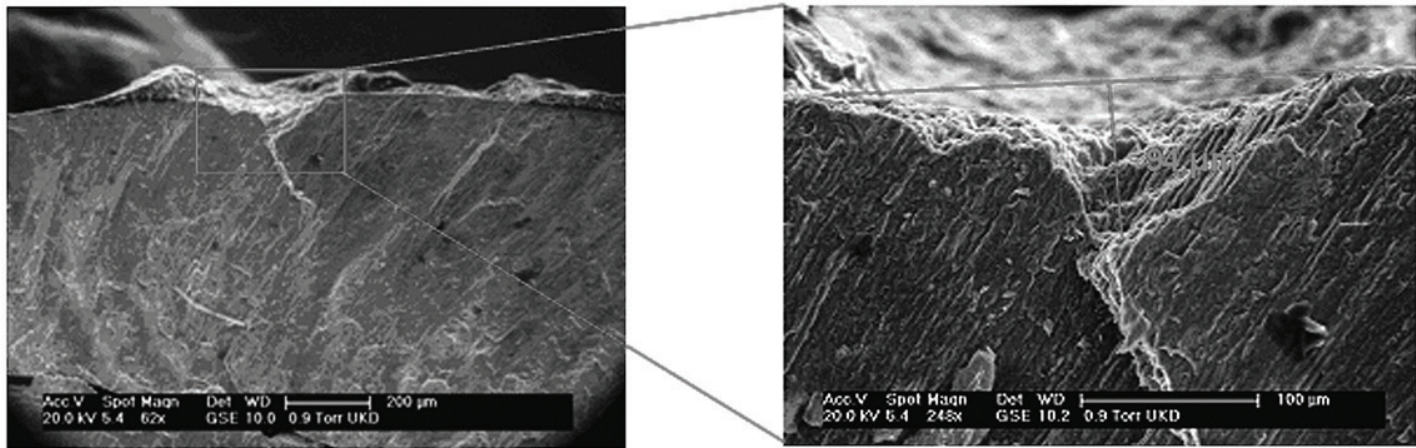

Fig 8: Enamel Tear-Out (a) Magnification $\times 62$, (b) Spot Magnification $\times 248$

\section{Clinical implication of cracks:}

- Risks of enamel cracks are more with ceramic brackets.

- Enamel cracks indicate that the bonding and debonding technique need improvement.

- There may be the need for pre-treatment examination of enamel cracks, notifying the patient beforehand if pronounced cracks are present.

\section{Enamel tearout}

Enamel tearouts may be related at least in part to the type of filler particles of the adhesive resins used for bonding and to the location of bond breakage. When comparisons were made between tooth surface appearances after debonding metal brackets attached with macrofilled (10$30 \mu \mathrm{m})$ and microfilled (0.2-0.3 $\mu \mathrm{m})$ adhesives, a difference occurred when the resin was scraped off with pliers. Small filler particles penetrated deep into the etched enamel to a greater degree as compared to macrofillers; so on debonding small filler particles reinforced the resin tags. However, macrofillers created a more natural break point in enamel-adhesive interface. With unfilled resins, there was no natural break point. Ceramic brackets using chemical retention caused enamel breakage more often than those using mechanical retention. ${ }^{25}$ (Figure 8)

\section{Residual adhesive removal}

To remove residual material, tungsten carbide burs are preferable to other methods like diamond burs, sandpaper disks, or rubber wheels. Water-cooling is recommended when bulk material is removed with high speed $(30,000$ rpm) to avoid pulp damage. When the last remnants of the adhesive are removed at a lower speed, better contrast between the adhesive and enamel is obtained without water-cooling. It has been shown that a carbide-finishing bur with a larger wedge angle and oblique ground chamfer produces less damage to the enamel surface than conventional carbide burs. ${ }^{26}$ Hosein et al pointed out that more surface enamel is lost during debonding and clean-up procedures than during bonding. ${ }^{27}$ Least enamel was lost during clean-up of brackets bonded with a self-etching primer system compared to conventional acid-etching technique. A tungsten carbide bur in a slow-speed handpiece and debonding pliers removed less enamel compared to high-speed handpiece and an ultrasonic scaler following conventional bonding procedures with phosphoric acid. Both ultrasonic scaler and high-speed tungsten bur resulted in as much as 25-30 $\mu \mathrm{m}$ loss of surface enamel. (Figure 9, 10) a
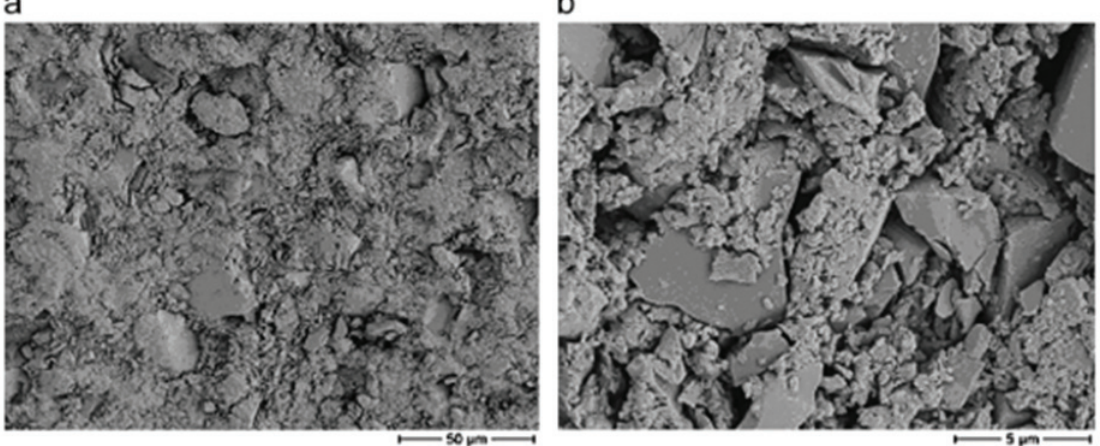

Figue 9: Adhesive remnants (a) magnification $\times 500$ and (b) spot magnification $\times 5000$

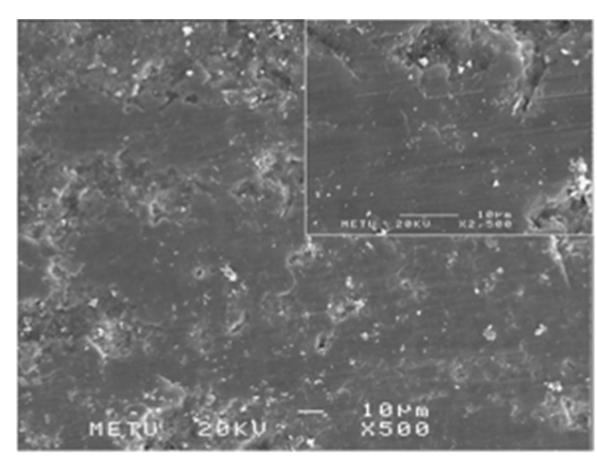

Figure 10: SEM analysis showing enamel surface after using tungsten carbide bur for cleaning the enamel surface 


\section{DISCUSSION}

The cumulative loss of enamel during cleaning, pumicing, etching, bonding and debonding might be less when compared to the thickness of enamel surface which is $1500-2000 \mu \mathrm{m}$. Only scarce research is available on the long-term effect of residual adhesive material on surface enamel. Resin tags can reach more than $20 \mu \mathrm{m}$ into the enamel after bonding based on acid-etching principle, and it can also alter the prism structure. Enamel damage can be considered as an inevitable sequelae to orthodontic treatment. Thus, every orthodontic practitioner should aim to minimize the damage to enamel, helping improve the longevity and beauty of teeth.

\section{REFERENCES}

1. Buonocore MG. A simple method of increasing the adhesion of acrylic filling materials to enamel surface. J Dent Res. 1955; 34:849-853.

2. Newman GV. Bonding plastic orthodontic attachments to tooth enamel. J NJ Dent Soc 1964; 35:346-358.

3. Retief DH, Dreyer CJ, Gavron G. The direct bonding of orthodontic attachments to teeth by means of an epoxy resin adhesive. Am J Orthod. 1970; 58:21-40.

4. Diedrich P: Enamel alterations from bracket bonding and debonding: a study with the scanning electron microscope. Am J Orthod. $1981 ; 79: 500-522$

5. Eliades T, Kakaboura A, Eliades G. Comparison of enamel colour changes associated with orthodontic bonding using two different adhesives. Eur J Orthod. 2001; 23:85-90.

6. Pus MD, Way DC. Enamel loss due to orthodontic bonding with filled and unfilled resins using various clean-up techniques. Am J Orthod. $1980 ; 79: 269-283$

7. Thompson RE, Way DC. Enamel loss due to prophylaxis and multiple bonding/debonding of orthodontic brackets. Am J Orthod. 1981; 79:282-295.

8. Fjeld $M, \varnothing$ gaard B. Scanning electron microscopic evaluation of enamel surfaces exposed to 3 orthodontic bonding systems. Am J Orthod Dentofacial Orthop. 2006; 130:575-581.

9. Miyazaki M, Hirohata N, Takagaki K. Influence of self-etching primer drying time on enamel bond strength of resin composites. J Dent. $1999 ; 27: 203-207$

10. Özcan M, Finnema K, Ybema A. Evaluation of failure characteristics and bond strength after ceramic and polycarbonate bracket debonding: Effect of bracket base silanization. Eur J Orthod. 2008; 30:176-82.

11. Ulusoyl Çaöry. Comparison of finishing and polishing systems for residual resin removal after debonding. J Appl Oral Sci. 2009; 17(3): 20915.

12. Ryf S, Flury S, Palaniappan S, Lussi A, VanMeerbeek B, Zimmerli B. Enamel loss and adhesive remnants following bracket removal and various clean-up procedures in vitro. Eur J Orthod. 2012; 34:25-32.

13. Swinburne ML, Willmot D, Patrick D. The use of debonding microspheres in electrothermal debonding. Eur J Orthod. 2011 ; $33: 407-12$.

14. Eliades T, Kakaboura A, Eliades G. Comparison of enamel colour changes associated with orthodontic bonding using two different adhesives. Eur J Orthod. 2001; 23:85-90.

15. Bishara S, Trulove T. Comparisons of different debonding techniques for ceramic brackets: an in vitro study. Part I. Background and methods. Am J Orthod Dento- facial Orthop. 1990; 98:145-153.

16. Bishara S, Trulove T. Comparisons of different debond- ing techniques for ceramic brackets: an in vitro study. Part II. Findings and clinical implications. Am J Orthod Dentofacial Orthop. 1990; 98:263-273.

17. Graber Vanarsdall. Orthodontics: Current principles \& techniques, 4th Ed. Ch. 14, p612-21.

18. Arici S, Minors C. The force levels required to mechanically debond ceramic brackets: an in vitro comparative study. Eur J Orthod. 2000; 22:327-334.

19. Bishara SE, Fonseca JM, Boyer DB: The use of debonding pliers in the removal of ceramic brackets: Force levels and enamel cracks. Am J Orthod Dentofacial Orthop. 1995; 108:242-248.

20. Mundstock KS, Sadowsky PL, Lacefield W. An in vitro evaluation of a metal reinforced orthodontic ceramic bracket. Am J Orthod Dentofacial Orthop. 1999; 116: 635-641.

21. Viazis AD, Cavanaugh G, Bevis RR. Bond strength of ceramic bracket under shear stress: An in vitro report, Am J Orthod. $1990 ; 98: 214$.

22. Reddy TB, Shivapuja PK; Debonding ceramic brackets: Effects on enamel. J Clin Orthod. 1991; 25:475.

23. Diedrich P. Enamel alterations from bracket bonding and debonding: a study with the scanning electron microscope. Am J Orthod. $1981 ; 79: 500-522$

24. Zachrisson BU, Skogan Ø, HØymyhr S. Enamel cracks in debonded, debanded and orthodontically untreated teeth. Am J Orthod. 1980; 77:307.

25. Bishara SE, Fehr DE. Ceramic brackets, something old, something new: A review. Semin Orthod 1997; 3:178.

26. Cambell PM. Enamel surfaces after orthodontic bracket debonding. Angle Orthod. 1995; 65:103-110.

27. Hosein I, Sherriff M, Ireland AJ. Enamel loss during bonding, debonding, and cleanup with use of self-etch- ing primer. Am J Orthod Dentofacial Orthop 2004; 126:717-724. 